\title{
Drug utilization and polypharmacy among the elderly: a survey in Rio de Janeiro City, Brazil
}

\author{
Suely Rozenfeld, ${ }^{1}$ Maria J. M. Fonseca, ${ }^{1}$ and Francisco A. Acurcio ${ }^{2}$
}

ABSTRACT Objectives. To describe drug utilization by Brazilian retirees with an emphasis on inappropriate use.

Methods. Cross-sectional study with a simple random sample of 800 retirees of the Brazilian Institute of Social Security, 60 years of age and older, residing in Rio de Janeiro City, through face-to-face interviews.

Results. Medication use in the 15 days before the study was reported by $85 \%$ of the sample, with a mean of 3.7 products per person (standard deviation $=2.9$ ). About half the sample used one to four medications and a third used five or more. The highest number of drugs used per patient was 24. More women than men used multiple medications. There is a tendency toward positive association $(P<0.001)$ between drug use and variables relating to disease and health care. This trend continues in the analysis by gender. Men who reported five or more diseases were five times more likely to use multiple drugs than men with up to two (zero, one, or two) diseases (prevalence ratio $5.21,95 \%$ confidence interval $=2.48-10.90$ ). Women who reported five or more diseases were nearly four times more likely to use multiple drugs than women with up to two diseases (prevalence ratio $3.67,95 \%$ confidence interval $=2.24-6.02$ ). Of the active substances used by the sample, $10 \%$ were considered inappropriate.

Conclusions. To improve drug therapy for the elderly, health practitioners can take measures to reduce unwarranted use of medication and to optimize the benefits from important drugs. Further studies should be conducted to adjust lists of medications inappropriate for the elderly to the situation in developing countries.

Key words Aging, health, drug utilization, polypharmacy, cross-sectional studies, Brazil.

The safety of pharmacotherapy for the elderly has been a concern since

\footnotetext{
National School of Public Health/Oswaldo Cruz Foundation, Rio de Janeiro, Rio de Janeiro, Brazil. Send correspondence and reprint requests to: Suely Rozenfeld, Rua Leopoldo Bulhões, 1480/8th floor, 21041-210 Rio de Janeiro, Rio de Janeiro, Brazil; telephone: 55-021-25982631; e-mail:rozenfel @ensp.fiocruz.br.

2 School of Pharmacy/Minas Gerais Federal University, Belo Horizonte, MG, Brazil.
}

the 1970s (1), and that concern has increased since the 1990s. Studies have pointed to disproportionate and inappropriate use of drugs with undesirable adverse reactions and their related costs. The quality of drug use was measured on quantitative criteria emphasizing the number of drugs and on qualitative criteria identifying the use of inappropriate medication (2). It is estimated that more than eight million office visits by the elderly in the United States resulted in a prescription for at least one inappropriate drug (3). One-fifth of the elderly in the United States took potentially inappropriate drugs (4). In Canada, analysis of drug prescriptions under a plan affording comprehensive drug coverage points in the same direction: $24 \%$ 
of the population between 65 and 74 years of age and $37 \%$ of people over age 75 were exposed to six or more different drugs during a 1-year period (5). This represents potential risks due to possible interactions and buildup of adverse events.

With the aging of the Brazilian population, use of drugs by the elderly is a growing concern. It poses challenges to the health care systems in addressing new diseases and therapeutics and in preventing and treating adverse events.

Some studies of drug use conducted in Brazil have included the elderly. They focused on the prevalence of drug use $(6,7)$ and on inappropriate prescriptions and use of drugs (8-10).

In 2002, the Brazilian Confederation for Retirees and Pensioners asked the Ministry of Health to undertake a study on drug consumption. The results of the study could help to improve the National Drug Policy, specifically with regard to drugrelated expenditure.

The purpose of the study is to describe drug use by retirees of the Brazilian Institute of Social Security who are 60 years of age or older and residing in the Municipality of Rio de Janeiro. Face-to-face home interviews were conducted that focused on polypharmacy and inappropriate drug use.

\section{MATERIALS AND METHODS}

This cross-sectional study drew on the database of the survey Uso de medicamentos por aposentados brasileiros (Medication use by Brazilian retirees), which interviewed elderly recipients of pension or retirement benefits granted by Brazil's National Social Security Institute. In December 2002, the base comprised data on 10446986 individuals 60 years old or older, 456628 of whom lived in Rio de Janeiro City. The probabilistic sample was selected considering a $95 \%$ significance level and a $75 \%$ response rate and assuming that no substantial differences exist between respondents and nonrespondents, which would permit sampling errors between $4 \%$ and $0.8 \%$ for prevalence rates between $50 \%$ and $1 \%$, respectively. Application of these criteria resulted in the selection of 800 individuals by simple random sampling, chosen from the register of beneficiaries held by Dataprev, the national agency responsible for processing social security information. An additional sample of 100 individuals was later included to replace those who had died, moved to other cities, or could not be located. The methodology is detailed in another paper (11).

Participants responded to questionnaires of closed, precodified questions about their social and demographic characteristics; self-rated health; diseases; health service use; private health coverage; and medication (products used, spending on medication, ability to afford products, need for assistance in taking medication).

Drug-related information was obtained by asking interviewees to bring with them the package, package insert, or prescription of any medication taken in the preceding 15 days, including vitamins and drugs for influenza and skin or eye conditions.

To facilitate identification of the active substances and to afford fuller understanding of drug use and related problems, the drug's brand name, manufacturer, pharmaceutical form, and period of use; how the drug was prescribed and acquired; and difficulties encountered in acquisition were also recorded.

Data were collected from March to August 2003 (autumn and winter). Parents or care givers were allowed to help participants respond to questions. The 19 interviewers were pharmacists or pharmacy students and were trained by the study coordinating team from whom they received written instructions. Interviewers' output was monitored weekly to prevent imbalances in the team and fraud. Data collection was also supervised directly; interviews were repeated in $10 \%$ subsamples and information was controlled statistically. Data entry operators were also trained. The data were input twice to enable errors to be detected and corrected.
Medications were identified and active substances were classified with the aid, respectively, of the Dictionary of pharmaceutical products (12) and the Anatomical Therapeutic Chemical Classification System (13).

Two units of analysis were used: the individual and the active substance. In describing sample characteristics and medication use by number of products, the unit of analysis was the individual. As use patterns differ substantially between men and women, the estimates are presented by sex. In describing the drugs, the unit of analysis was the active substance.

Two approaches were used to examine the suitability of drug therapy in this sample of elderly people: the concept of polypharmacy (use of five or more products at the same time) and the internationally proposed lists of active substances that are inappropriate for the elderly. One point of departure was Fick et al. (2), whose list was adapted for this study to the substances found in products available in Brazil. The concept of polypharmacy was explored with three variables. The first variable involved the following categories of drug use: none, one to four, and five or more; the second variable included the categories "no" and "yes"; and the third variable included the categories one to four and five or more.

The differences among these categories are presented as proportions, and their significance was tested with the Pearson $\chi^{2}$ test and the test of tendency. A value of $P<0.05$ was used.

The response rate to the home interviews was $70.7 \%$ (11). The age of six interviewees was not known and was estimated according to ages in the Dataprev register.

\section{RESULTS}

Medication had been used in the 15 days before the interview by $85 \%$ of the interviewees (490 of 577). An average of 3.7 products were used per person (standard deviation $=2.9$ ). No help was needed to take medication by 
$78.3 \%$ (471) of the users. Of the 2112 drugs used by the elderly, $93.5 \%$ (1975) had been prescribed by physicians. Of the drugs used, $4 \%$ were manufactured by government laboratories, and $75 \%$ of those were manufactured by Farmanguinhos/Oswaldo Cruz Foundation.

Table 1 presents data from the individuals interviewed. Of these individuals, $41.1 \%$ were less than 70 years old, $38.1 \%$ had 8 years or more of schooling, $84.7 \%$ lived in their own homes, and $18.4 \%$ lived alone. Also, $46.2 \%$ self-rated their health condition as good or very good, $43.1 \%$ had private health coverage, and $77.1 \%$ reported up to four diseases. In the previous year, $89.4 \%$ were not confined to bed, $49.6 \%$ made one to five office visits to physicians, and $13.9 \%$ were hospitalized. At the interview, $17 \%$ reported diabetes, $59.9 \%$ had hypertension, and $42.6 \%$ had bone and articular diseases.

There were some differences $(P<$ $0.001)$ between males and females: more years of education among men; more women living alone; and a higher proportion of women with worse self-rated health conditions, with more office visits to physicians, and with bone and articular diseases. Also, $11 \%$ of the women and $21 \%$ of the men had used no medication in the 15 days before the interview.

Table 2 displays the distribution of 2466 active substances by the main groups (first level) and pharmacologic/therapeutic subgroups (second level) from the Anatomical Therapeutic Chemical Classification. The most commonly used active substances were cardiovascular agents $(34.4 \%)$, alimentary tract and metabolism agents $(20.5 \%)$, and central nervous system agents $(15.5 \%)$. The most consumed subgroups, in descending order, are diuretics, agents acting on the renin-angiotensin system, and calcium-channel blockers (cardiovascular system); vitamins, drugs used in diabetes, antacids, and drugs for treatment of peptic ulcer and flatulence (alimentary tract and metabolism); and analgesics, psychoanaleptics, and psycholeptics (nervous system).

TABLE 1. Characteristics of retirees 60 years old or older, Rio de Janeiro City, Rio de Janeiro, Brazil, $2003(n=577)$

\begin{tabular}{|c|c|c|c|c|c|c|}
\hline \multirow[b]{2}{*}{ Variable } & \multicolumn{2}{|c|}{ Men } & \multicolumn{2}{|c|}{ Women } & \multicolumn{2}{|c|}{ Total } \\
\hline & No. & $\%$ & No. & $\%$ & No. & $\%$ \\
\hline \multicolumn{7}{|l|}{ Age group (years) } \\
\hline $60-69$ & 94 & 41.8 & 143 & 40.6 & 237 & 41.1 \\
\hline $70-79$ & 97 & 43.1 & 134 & 38.1 & 231 & 40.0 \\
\hline$\geq 80$ & 34 & 15.1 & 75 & 21.3 & 109 & 18.9 \\
\hline \multicolumn{7}{|l|}{ Education $^{\mathrm{a}}$} \\
\hline Illiterate & 18 & 8.0 & 46 & 13.1 & 64 & 11.1 \\
\hline Some elementary school & 100 & 44.4 & 192 & 54.9 & 292 & 50.8 \\
\hline Elementary school & 33 & 14.7 & 49 & 14.0 & 82 & 14.3 \\
\hline High school degree & 41 & 18.2 & 46 & 13.1 & 87 & 15.1 \\
\hline College degree & 33 & 14.7 & 17 & 4.9 & 50 & 8.7 \\
\hline \multicolumn{7}{|l|}{ Household } \\
\hline Own & 195 & 86.7 & 293 & 83.2 & 488 & 84.7 \\
\hline Rented & 16 & 7.1 & 35 & 9.9 & 51 & 8.9 \\
\hline Other & 14 & 6.2 & 24 & 6.9 & 37 & 6.4 \\
\hline \multicolumn{7}{|l|}{ Number in household ${ }^{b}$} \\
\hline Lives alone & 30 & 13.3 & 76 & 21.7 & 106 & 18.4 \\
\hline Lives with spouse/siblings & 182 & 80.9 & 218 & 62.1 & 400 & 69.4 \\
\hline Lives with others & 13 & 5.8 & 57 & 16.2 & 70 & 12.2 \\
\hline \multicolumn{7}{|l|}{ Self-reported health ${ }^{\mathrm{a}}$} \\
\hline Very good & 34 & 15.9 & 30 & 9.8 & 64 & 11.5 \\
\hline Good & 83 & 39.0 & 109 & 31.9 & 192 & 34.7 \\
\hline Fair & 81 & 38.0 & 160 & 46.9 & 241 & 43.5 \\
\hline Poor & 10 & 4.7 & 28 & 8.3 & 38 & 6.9 \\
\hline Very poor & 5 & 2.4 & 14 & 4.1 & 19 & 3.4 \\
\hline \multicolumn{7}{|l|}{ Confined to bed in the last year } \\
\hline Yes & 22 & 9.8 & 39 & 11.1 & 61 & 10.6 \\
\hline No & 203 & 90.2 & 313 & & 516 & 89.4 \\
\hline \multicolumn{7}{|l|}{ Office visits to physicians ${ }^{a}$} \\
\hline None & 31 & 13.8 & 28 & 8.0 & 59 & 10.3 \\
\hline 1 to 5 & 122 & 54.2 & 163 & 46.7 & 285 & 49.6 \\
\hline$\geq 6$ & 72 & 32.0 & 158 & 45.3 & 230 & 40.1 \\
\hline \multicolumn{7}{|l|}{ Hospitalized last year } \\
\hline Yes & 37 & 16.4 & 42 & 12.0 & 79 & 13.9 \\
\hline No & 188 & 83.6 & 307 & 88.0 & 495 & 86.1 \\
\hline \multicolumn{7}{|l|}{ Private Health Coverage } \\
\hline Yes & 104 & 46.2 & 144 & 41.1 & 248 & 43.1 \\
\hline No & 121 & 53.8 & 206 & 58.9 & 327 & 56.9 \\
\hline \multicolumn{7}{|l|}{ Number of diseases ${ }^{a}$} \\
\hline None & 19 & 8.4 & 11 & 3.1 & 30 & 5.2 \\
\hline 1 or 2 & 78 & 34.7 & 93 & 26.5 & 171 & 29.7 \\
\hline 3 or 4 & 92 & 40.9 & 151 & 43.0 & 243 & 42.2 \\
\hline$\geq 5$ & 36 & 16.0 & 96 & 27.4 & 132 & 22.9 \\
\hline \multicolumn{7}{|l|}{ Diabetes } \\
\hline Yes & 32 & 14.2 & 66 & 18.9 & 98 & 17.0 \\
\hline No & 193 & 85.8 & 284 & 81.1 & 477 & 83.0 \\
\hline \multicolumn{7}{|l|}{ Hypertension } \\
\hline Yes & 125 & 55.6 & 220 & 62.7 & 345 & 59.9 \\
\hline No & 100 & 44.4 & 131 & 37.3 & 231 & 40.1 \\
\hline \multicolumn{7}{|l|}{ Bone and articular diseases ${ }^{b}$} \\
\hline Yes & 61 & 27.4 & 183 & 52.3 & 244 & 42.6 \\
\hline No & 162 & 72.6 & 167 & 47.7 & 329 & 57.4 \\
\hline
\end{tabular}

a Linear trend $\chi^{2}(P<0.001)$.

b Pearson $\chi^{2}(P<0.001)$

The association between use of medication and health condition and health service use was examined in two ways: by stratifying the variable medication use into three strata by the number of products used (none, one to four, five or more) and into two strata (no use versus use; four versus five or 
TABLE 2. Distribution of active substances used by retirees 60 years old or more, according to Anatomical Therapeutic Chemical Classification, Rio de Janeiro City, Rio de Janeiro, Brazil, 2003 ( $n=2$ 466)

\begin{tabular}{|c|c|c|c|}
\hline Anatomical Therapeutic Chemical Classification & Frequency & $\begin{array}{l}\text { Percentage } \\
\text { within } \\
\text { subgroups }^{a}\end{array}$ & $\begin{array}{l}\text { Percentage } \\
\text { within } \\
\text { total }^{\mid \mathrm{a}}\end{array}$ \\
\hline $\begin{array}{l}\text { A Alimentary tract and metabolism } \\
\text { A02 Drugs for acid-related disorders } \\
\text { A10 Drugs used in diabetes } \\
\text { A11 Vitamins } \\
\text { A12 Mineral supplements }\end{array}$ & $\begin{array}{r}506 \\
74 \\
96 \\
201 \\
66\end{array}$ & $\begin{array}{r}100 \\
14.6 \\
19.0 \\
39.7 \\
13.0\end{array}$ & $\begin{array}{r}20.5 \\
3.0 \\
3.9 \\
8.1 \\
2.7\end{array}$ \\
\hline $\begin{array}{l}\text { B Blood and blood-forming organs } \\
\text { B01 Antithrombotic agents } \\
\text { B02 Antihemorrhagics }\end{array}$ & $\begin{array}{r}182 \\
116 \\
49\end{array}$ & $\begin{array}{r}100 \\
63.7 \\
26.9\end{array}$ & $\begin{array}{l}7.4 \\
4.7 \\
2.0\end{array}$ \\
\hline $\begin{array}{l}\text { C Cardiovascular system } \\
\text { C01 Cardiac therapy } \\
\text { C03 Diuretics } \\
\text { C07 Beta-blocking agents } \\
\text { C08 Calcium-channel blockers } \\
\text { C09 Agents acting on the renin-angiotensin system }\end{array}$ & $\begin{array}{r}848 \\
95 \\
227 \\
92 \\
117 \\
202\end{array}$ & $\begin{array}{r}100 \\
11.2 \\
26.2 \\
10.8 \\
13.8 \\
23.8\end{array}$ & $\begin{array}{r}34.4 \\
3.8 \\
9.2 \\
3.7 \\
4.7 \\
8.2\end{array}$ \\
\hline $\begin{array}{l}\text { D Dermatologicals } \\
\text { D01 Antifungals for dermatological use } \\
\text { D06 Antibiotics and chemotherapeutics for } \\
\text { dermatological use } \\
\text { D07 Corticosteroids, dermatological preparations } \\
\text { D08 Antiseptics and disinfectants }\end{array}$ & $\begin{array}{r}13 \\
21 \\
9\end{array}$ & $\begin{array}{l}17.8 \\
28.8 \\
12.3\end{array}$ & $\begin{array}{l}0.5 \\
0.8 \\
0.4\end{array}$ \\
\hline $\begin{array}{l}\text { G Genitourinary system and sex hormones } \\
\text { G03 Sex hormones and modulators of the genital } \\
\text { system } \\
\text { G04 Urologicals }\end{array}$ & $\begin{array}{r}29 \\
18 \\
7\end{array}$ & $\begin{array}{l}100 \\
62.1 \\
24.1\end{array}$ & $\begin{array}{l}1.2 \\
0.7 \\
0.3\end{array}$ \\
\hline $\begin{array}{l}\text { H Systemic hormonal preparations, excluding } \\
\text { sex hormones and insulin } \\
\text { H02 Corticosteroids for systemic use } \\
\text { H03 Thyroid therapy }\end{array}$ & $\begin{array}{r}24 \\
9 \\
15\end{array}$ & $\begin{array}{r}100 \\
37.5 \\
62.5\end{array}$ & $\begin{array}{l}1.0 \\
0.6 \\
0.4\end{array}$ \\
\hline $\begin{array}{l}\text { J Anti-infectives for systemic use } \\
\text { J01 Antibacterials for systemic use }\end{array}$ & $\begin{array}{l}47 \\
44\end{array}$ & $\begin{array}{r}100 \\
93.6\end{array}$ & $\begin{array}{l}1.9 \\
1.8\end{array}$ \\
\hline $\begin{array}{l}\text { L Antineoplastic and immunomodulating agents } \\
\text { L02 Endocrine therapy } \\
\text { L04 Immunosuppressive agents }\end{array}$ & $\begin{array}{l}5 \\
3 \\
2\end{array}$ & $\begin{array}{l}100 \\
60.0 \\
40.0\end{array}$ & $\begin{array}{l}0.2 \\
0.1 \\
0.1\end{array}$ \\
\hline $\begin{array}{l}\text { M Musculoskeletal system } \\
\text { M01 Anti-inflammatory and antirheumatic products } \\
\text { M03 Muscle relaxants } \\
\text { M05 Drugs for treatment of bone diseases }\end{array}$ & $\begin{array}{r}154 \\
96 \\
23 \\
14\end{array}$ & $\begin{array}{r}100 \\
62.3 \\
14.9 \\
9.1\end{array}$ & $\begin{array}{l}6.2 \\
3.9 \\
0.9 \\
0.6\end{array}$ \\
\hline $\begin{array}{l}\text { N Nervous system } \\
\text { N02 Analgesics } \\
\text { N05 Psycholeptics } \\
\text { N06 Psychoanaleptics } \\
\text { N07 Other nervous system drugs }\end{array}$ & $\begin{array}{r}383 \\
104 \\
92 \\
98 \\
32\end{array}$ & $\begin{array}{r}100 \\
27.2 \\
24.0 \\
25.6 \\
8.4\end{array}$ & $\begin{array}{r}15.5 \\
4.2 \\
3.7 \\
3.9 \\
1.3\end{array}$ \\
\hline $\begin{array}{l}\text { P Antiparasitic products, insecticides, and repellents } \\
\text { P01 Antiprotozoals } \\
\text { P02 Anthelmintics }\end{array}$ & $\begin{array}{l}6 \\
2 \\
3\end{array}$ & $\begin{array}{r}100 \\
33.3 \\
50.0\end{array}$ & $\begin{array}{l}0.2 \\
0.1 \\
0.1\end{array}$ \\
\hline $\begin{array}{l}\text { R Respiratory system } \\
\text { R03 Drugs for obstructive airway diseases } \\
\text { R05 Cough and cold preparations } \\
\text { R06 Antihistamines for systemic use }\end{array}$ & $\begin{array}{l}75 \\
20 \\
13 \\
30\end{array}$ & $\begin{array}{r}100 \\
26.7 \\
17.3 \\
40.0\end{array}$ & $\begin{array}{l}3.0 \\
0.8 \\
0.5 \\
1.2\end{array}$ \\
\hline $\begin{array}{l}\text { S Sensory organs } \\
\text { S01 Ophthalmologicals } \\
\text { S02 Otologicals }\end{array}$ & $\begin{array}{r}131 \\
116 \\
8\end{array}$ & $\begin{array}{r}100 \\
88.5 \\
6.1\end{array}$ & $\begin{array}{l}5.3 \\
4.7 \\
0.3\end{array}$ \\
\hline $\begin{array}{l}\text { V Various } \\
\text { V03 All other therapeutic products }\end{array}$ & $\begin{array}{l}03 \\
03\end{array}$ & $\begin{array}{l}100 \\
100\end{array}$ & $\begin{array}{l}0.1 \\
0.1\end{array}$ \\
\hline
\end{tabular}

a The most frequent groups and subgroups are presented here. The drugs specified in each subgroup represent at least $80 \%$ of the group total. more). The results are shown in Tables 3,4 , and 5 .

About half the sample (52.2\%) used one to four medications and about a third $(32.7 \%)$ used five or more. Multiple medication use was found in a larger proportion of women than men: $37.2 \%$ of women and $25.8 \%$ of men used five or more products. The tendency to use a larger number of medications is greater among those who have more schooling, own their homes, live with other people, or are more elderly. However, only the association with age among men is statistically significant $(P=0.027)$ (Table 3$)$.

Table 4 shows that the number of medications used increases significantly $(P<0.05)$ among those who self-rate their health as worse; are confined to bed; have made more office visits to physicians; report a larger number of illnesses; or have diabetes, hypertension, or osteoarticular diseases. These results are consistent with those found when the variable "use of medications" is categorized into two strata (Table 5). The consistency also holds in analysis of the relationship of "has private health coverage" and "has been hospitalized in the previous 12 months" with the variable "use of medications": there is no statistically significant association, regardless of whether two or three strata of the variable "use of medications" are applied (Table 5).

Table 6 indicates the active substances potentially inappropriate for older adults and used by the sample. They correspond to $10.4 \%$ (256/2 466) of all the active substances recorded. Muscle relaxants, antihistamines, and long-acting benzodiazepines were the most common.

\section{DISCUSSION}

This study examined the pattern of medication use among the elderly in Rio de Janeiro. The sample, comprising social insurance retirees, thus consisted of relatively elderly people with greater access to health services. Of the women interviewed, a larger proportion had more schooling and lived 
TABLE 3. Prevalence of drug use (three strata) according to social and demographic variables, among retirees 60 years old or older, Rio de Janeiro City, Rio de Janeiro, Brazil, 2003

\begin{tabular}{|c|c|c|c|c|c|c|c|c|c|}
\hline \multirow[b]{2}{*}{ Variable } & \multicolumn{3}{|c|}{ No. of men (\%) } & \multicolumn{3}{|c|}{ No. of women (\%) } & \multicolumn{3}{|c|}{ Total number (\%) } \\
\hline & 0 & $1-4$ & $\geq 5$ & 0 & $1-4$ & $\geq 5$ & 0 & $1-4$ & $\geq 5$ \\
\hline \multicolumn{10}{|l|}{ Age group ${ }^{a}$} \\
\hline $60-69$ & $\begin{array}{c}26 \\
(27.7)\end{array}$ & $\begin{array}{c}48 \\
(51.1)\end{array}$ & $\begin{array}{c}20 \\
(21.3)\end{array}$ & $\begin{array}{c}16 \\
(11.2)\end{array}$ & $\begin{array}{c}77 \\
(53.8)\end{array}$ & $\begin{array}{c}50 \\
(35.0)\end{array}$ & $\begin{array}{c}42 \\
(17.7)\end{array}$ & $\begin{array}{c}125 \\
(52.7)\end{array}$ & $\begin{array}{c}70 \\
(29.5)\end{array}$ \\
\hline $70-79$ & $\begin{array}{c}21 \\
(21.6)\end{array}$ & $\begin{array}{c}47 \\
(48.5)\end{array}$ & $\begin{array}{c}29 \\
(29.9)\end{array}$ & $\begin{array}{c}14 \\
(10.4)\end{array}$ & $\begin{array}{c}70 \\
(52.2)\end{array}$ & $\begin{array}{c}50 \\
(37.3)\end{array}$ & $\begin{array}{c}35 \\
(15.2)\end{array}$ & $\begin{array}{c}117 \\
(50.6)\end{array}$ & $\begin{array}{c}79 \\
(34.2)\end{array}$ \\
\hline$\geq 80$ & $\begin{array}{c}1 \\
(2.9)\end{array}$ & $\begin{array}{c}24 \\
(70.6)\end{array}$ & $\begin{array}{c}9 \\
(26.5)\end{array}$ & $\begin{array}{c}9 \\
(12.0)\end{array}$ & $\begin{array}{c}35 \\
(46.7)\end{array}$ & $\begin{array}{c}31 \\
(41.3)\end{array}$ & $\begin{array}{c}10 \\
(9.2)\end{array}$ & $\begin{array}{c}59 \\
(54.1)\end{array}$ & $\begin{array}{c}40 \\
(36.7)\end{array}$ \\
\hline \multicolumn{10}{|l|}{ Education } \\
\hline Some elementary school & $\begin{array}{c}31 \\
(26.3)\end{array}$ & $\begin{array}{c}59 \\
(50.0)\end{array}$ & $\begin{array}{c}28 \\
(23.7)\end{array}$ & $\begin{array}{c}29 \\
(12.2)\end{array}$ & $\begin{array}{c}125 \\
(52.5)\end{array}$ & $\begin{array}{c}84 \\
(35.3)\end{array}$ & $\begin{array}{c}60 \\
(16.9)\end{array}$ & $\begin{array}{c}184 \\
(51.7)\end{array}$ & $\begin{array}{c}112 \\
(31.5)\end{array}$ \\
\hline Elementary school or more & $\begin{array}{c}17 \\
(15.9)\end{array}$ & $\begin{array}{c}60 \\
(56.1)\end{array}$ & $\begin{array}{c}30 \\
(28.0)\end{array}$ & $\begin{array}{c}10 \\
(8.9)\end{array}$ & $\begin{array}{c}56 \\
(50.0)\end{array}$ & $\begin{array}{c}46 \\
(41.1)\end{array}$ & $\begin{array}{c}27 \\
(12.3)\end{array}$ & $\begin{array}{c}116 \\
(53.0)\end{array}$ & $\begin{array}{c}76 \\
(34.7)\end{array}$ \\
\hline \multicolumn{10}{|l|}{ Household } \\
\hline Not own & $\begin{array}{c}11 \\
(37.9)\end{array}$ & $\begin{array}{c}12 \\
(41.4)\end{array}$ & $\begin{array}{c}6 \\
(20.7)\end{array}$ & $\begin{array}{c}6 \\
(10.6)\end{array}$ & $\begin{array}{c}28 \\
(47.5)\end{array}$ & $\begin{array}{c}25 \\
(42.4)\end{array}$ & $\begin{array}{c}17 \\
(19.3)\end{array}$ & $\begin{array}{c}40 \\
(45.5)\end{array}$ & $\begin{array}{c}31 \\
(35.2)\end{array}$ \\
\hline Own & $\begin{array}{c}36 \\
(18.5)\end{array}$ & $\begin{array}{c}107 \\
(54.9)\end{array}$ & $\begin{array}{c}52 \\
(26.7)\end{array}$ & $\begin{array}{c}33 \\
(11.3)\end{array}$ & $\begin{array}{c}154 \\
(52.6)\end{array}$ & $\begin{array}{c}106 \\
(36.2)\end{array}$ & $\begin{array}{c}69 \\
(14.1)\end{array}$ & $\begin{array}{c}261 \\
(53.5)\end{array}$ & $\begin{array}{c}158 \\
(32.4)\end{array}$ \\
\hline \multicolumn{10}{|l|}{ Number in household } \\
\hline Lives with others & $\begin{array}{c}37 \\
(19.0)\end{array}$ & $\begin{array}{c}105 \\
(53.8)\end{array}$ & $\begin{array}{c}53 \\
(27.2)\end{array}$ & $\begin{array}{c}29 \\
(10.5)\end{array}$ & $\begin{array}{c}142 \\
(51.6)\end{array}$ & $\begin{array}{c}104 \\
(37.8)\end{array}$ & $\begin{array}{c}66 \\
(14.0)\end{array}$ & $\begin{array}{c}247 \\
(52.6)\end{array}$ & $\begin{array}{c}157 \\
(33.4)\end{array}$ \\
\hline Lives alone & $\begin{array}{c}11 \\
(36.7)\end{array}$ & $\begin{array}{c}14 \\
(46.7)\end{array}$ & $\begin{array}{c}5 \\
(16.7)\end{array}$ & $\begin{array}{c}10 \\
(13.2)\end{array}$ & $\begin{array}{c}40 \\
(52.6)\end{array}$ & $\begin{array}{c}26 \\
(34.2)\end{array}$ & $\begin{array}{c}21 \\
(19.8)\end{array}$ & $\begin{array}{c}54 \\
(50.9)\end{array}$ & $\begin{array}{c}31 \\
(29.2)\end{array}$ \\
\hline
\end{tabular}

alone than the men; they also selfrated their health condition as worse.

At interview, participants were asked to present the prescriptions, packages, or patient information inserts of whatever pharmaceuticals they were using. This was done to facilitate identification of the medications and to improve the validity information on use. Prescriptions, packages, or patient information inserts were presented for most of the products. In addition, $94 \%$ of the products were reported by interviewees as having been prescribed by a doctor. This does not mean, however, that those products are sold exclusively on presentation of a doctor's prescription. In Brazil, it is common for the same prescription to be presented several times to acquire medications (14).

Medication use in the 15 days before the study was reported by $85 \%$ of the sample; the mean number of products used was 3.7 (standard deviation = 2.9). Women used more products, which agrees with data from interna- tional studies (15). The groups of medications most used are cardiovascular, alimentary tract and metabolism, and central nervous system drugs, coinciding with findings of other Brazilian studies $(6,7,9,10)$; however, the prevalence of use and the number of drugs used were slightly lower than in other studies, ranging from $72 \%$ to $84 \%$, with the mean number of products used per person ranging between 2.2 and 3.2. These discrepancies may reflect differences in consumption among the populations studied. However, differences among sample selection criteria, whether population based $(6,10)$ or not $(7,9)$, and among recall periods in these studies may partly explain the differences found between their results and ours.

We assessed the quality of medication use in two ways: in quantitative descriptions (polypharmacy), where the emphasis is on the number of medications, and in a qualitative description, which focused on the appropriateness of the substances used.

\section{Polypharmacy}

In our study, the likelihood of more than one product being used increased with age, with improved social and economic conditions, and with a worsening state of health. For example, men who reported five or more diseases were at a five times higher risk of being multiple users than men with up to two (zero, one, or two) diseases (prevalence ratio 5.21, 95\% confidence interval $=2.48-10.9$ ). Among women with five or more diseases, the likelihood of being a multiple user was nearly four times greater than for women with up to two diseases (prevalence ratio $3.67,95 \%$ confidence interval $=2.24-6.02)$.

One review paper (16) estimated the proportion of noninstitutionalized elderly people exposed to more than five products at between $5 \%$ and $13 \%$. Individual studies found use rates of five or more products in excess of $25 \%$ (17) or even higher, above $44 \%$ (15). Being female is considered an inde- 
TABLE 4. Prevalence of drug use (three strata) by health condition and health service variables, among retirees 60 years old or older, Rio de Janeiro City, Rio de Janeiro, Brazil, 2003

\begin{tabular}{|c|c|c|c|c|c|c|c|c|c|}
\hline \multirow[b]{2}{*}{ Variable } & \multicolumn{3}{|c|}{ No. of males (\%) } & \multicolumn{3}{|c|}{ No. of females (\%) } & \multicolumn{3}{|c|}{ Total number (\%) } \\
\hline & 0 & $1-4$ & $\geq 5$ & 0 & $1-4$ & $\geq 5$ & 0 & $1-4$ & $\geq 5$ \\
\hline \multicolumn{10}{|l|}{ Self-reported health ${ }^{a}$} \\
\hline Very good/good & $\begin{array}{c}35 \\
(29.9)\end{array}$ & $\begin{array}{c}67 \\
(57.3)\end{array}$ & $\begin{array}{c}15 \\
(12.8)\end{array}$ & $\begin{array}{c}26 \\
(18.7)\end{array}$ & $\begin{array}{c}84 \\
(60.4)\end{array}$ & $\begin{array}{c}29 \\
(20.9)\end{array}$ & $\begin{array}{c}61 \\
(23.8)\end{array}$ & $\begin{array}{c}151 \\
(59.0)\end{array}$ & $\begin{array}{c}44 \\
(17.2)\end{array}$ \\
\hline Fair & $\begin{array}{c}12 \\
(14.8)\end{array}$ & $\begin{array}{c}39 \\
(48.1)\end{array}$ & $\begin{array}{c}30 \\
(37.0)\end{array}$ & $\begin{array}{c}9 \\
(5.6)\end{array}$ & $\begin{array}{c}76 \\
(47.5)\end{array}$ & $\begin{array}{c}75 \\
(46.9)\end{array}$ & $\begin{array}{l}21 \\
(8.7)\end{array}$ & $\begin{array}{c}115 \\
(47.7)\end{array}$ & $\begin{array}{c}105 \\
(43.6)\end{array}$ \\
\hline Very poor/poor & 0 & $\begin{array}{c}6 \\
(40.0)\end{array}$ & $\begin{array}{c}9 \\
(60.0)\end{array}$ & $\begin{array}{c}3 \\
(7.1)\end{array}$ & $\begin{array}{c}18 \\
(42.9)\end{array}$ & $\begin{array}{c}21 \\
(50.0)\end{array}$ & $\begin{array}{c}3 \\
(5.3)\end{array}$ & $\begin{array}{c}24 \\
(42.1)\end{array}$ & $\begin{array}{c}30 \\
(52.8)\end{array}$ \\
\hline \multicolumn{10}{|l|}{ Confined to bed ${ }^{a}$} \\
\hline No & $\begin{array}{c}45 \\
(22.2)\end{array}$ & $\begin{array}{c}111 \\
(54.7)\end{array}$ & $\begin{array}{c}47 \\
(23.2)\end{array}$ & $\begin{array}{c}37 \\
(11.8)\end{array}$ & $\begin{array}{c}167 \\
(53.4)\end{array}$ & $\begin{array}{c}109 \\
(34.8)\end{array}$ & $\begin{array}{c}82 \\
(15.2)\end{array}$ & $\begin{array}{c}278 \\
(53.9)\end{array}$ & $\begin{array}{c}156 \\
(30.2)\end{array}$ \\
\hline Yes & $\begin{array}{c}3 \\
(13.6)\end{array}$ & $\begin{array}{c}8 \\
(36.4)\end{array}$ & $\begin{array}{c}11 \\
(50.0)\end{array}$ & $\begin{array}{c}2 \\
(5.1)\end{array}$ & $\begin{array}{c}15 \\
(38.5)\end{array}$ & $\begin{array}{c}22 \\
(56.4)\end{array}$ & $\begin{array}{c}5 \\
(8.2)\end{array}$ & $\begin{array}{c}23 \\
(37.7)\end{array}$ & $\begin{array}{c}33 \\
(54.1)\end{array}$ \\
\hline \multicolumn{10}{|l|}{ Office visits ${ }^{a}$} \\
\hline $0-5$ & $\begin{array}{c}46 \\
(30.1)\end{array}$ & $\begin{array}{c}87 \\
(56.9)\end{array}$ & $\begin{array}{c}20 \\
(13.1)\end{array}$ & $\begin{array}{c}34 \\
(17.8)\end{array}$ & $\begin{array}{c}112 \\
(58.6)\end{array}$ & $\begin{array}{c}45 \\
(23.6)\end{array}$ & $\begin{array}{c}80 \\
(23.3)\end{array}$ & $\begin{array}{c}199 \\
(57.8)\end{array}$ & $\begin{array}{c}65 \\
(18.9)\end{array}$ \\
\hline$\geq 6$ & $\begin{array}{c}2 \\
(2.8)\end{array}$ & $\begin{array}{c}32 \\
(44.4)\end{array}$ & $\begin{array}{c}38 \\
(52.8)\end{array}$ & $\begin{array}{c}4 \\
(2.5)\end{array}$ & $\begin{array}{c}68 \\
(43.0)\end{array}$ & $\begin{array}{c}86 \geq 6 \\
(54.4)\end{array}$ & $\begin{array}{c}6 \\
(2.6)\end{array}$ & $\begin{array}{c}100 \\
(43.5)\end{array}$ & $\begin{array}{c}124 \\
(53.9)\end{array}$ \\
\hline \multicolumn{10}{|l|}{ Hospitalized ${ }^{b}$} \\
\hline No & $\begin{array}{c}43 \\
(22.9)\end{array}$ & $\begin{array}{c}102 \\
(54.3)\end{array}$ & $\begin{array}{c}43 \\
(22.9)\end{array}$ & $\begin{array}{c}39 \\
(12.7)\end{array}$ & $\begin{array}{c}163 \\
(53.1)\end{array}$ & $\begin{array}{c}105 \\
(34.2)\end{array}$ & $\begin{array}{c}82 \\
(16.6)\end{array}$ & $\begin{array}{c}265 \\
(53.5)\end{array}$ & $\begin{array}{c}148 \\
(29.9)\end{array}$ \\
\hline Yes & $\begin{array}{c}5 \\
(13.5)\end{array}$ & $\begin{array}{c}17 \\
(45.9)\end{array}$ & $\begin{array}{c}15 \\
(40.5)\end{array}$ & 0 & $\begin{array}{c}16 \\
(38.1)\end{array}$ & $\begin{array}{c}26 \\
(61.9)\end{array}$ & $\begin{array}{c}5 \\
(6.3)\end{array}$ & $\begin{array}{c}33 \\
(41.8)\end{array}$ & $\begin{array}{c}41 \\
(51.9)\end{array}$ \\
\hline \multicolumn{10}{|c|}{ Private health coverage ${ }^{c}$} \\
\hline No & $\begin{array}{c}31 \\
(25.6)\end{array}$ & $\begin{array}{c}64 \\
(52.9)\end{array}$ & $\begin{array}{c}26 \\
(21.5)\end{array}$ & $\begin{array}{c}26 \\
(12.6)\end{array}$ & $\begin{array}{c}111 \\
(53.9)\end{array}$ & $\begin{array}{c}69 \\
(33.5)\end{array}$ & $\begin{array}{c}57 \\
(17.4)\end{array}$ & $\begin{array}{c}175 \\
(53.5)\end{array}$ & $\begin{array}{c}95 \\
(29.1)\end{array}$ \\
\hline Yes & $\begin{array}{c}17 \\
(16.3)\end{array}$ & $\begin{array}{c}55 \\
(52.9)\end{array}$ & $\begin{array}{c}32 \\
(30.8)\end{array}$ & $\begin{array}{c}13 \\
(9.0)\end{array}$ & $\begin{array}{c}69 \\
(47.9)\end{array}$ & $\begin{array}{c}62 \\
(43.1)\end{array}$ & $\begin{array}{c}30 \\
(12.1)\end{array}$ & $\begin{array}{c}124 \\
(50.0)\end{array}$ & $\begin{array}{c}94 \\
(37.9)\end{array}$ \\
\hline \multicolumn{10}{|l|}{ Number of diseases ${ }^{a}$} \\
\hline $0-2$ & $\begin{array}{c}39 \\
(40.2)\end{array}$ & $\begin{array}{c}51 \\
(52.6)\end{array}$ & $\begin{array}{c}7 \\
(7.2)\end{array}$ & $\begin{array}{c}28 \\
(26.9)\end{array}$ & $\begin{array}{c}62 \\
(59.6)\end{array}$ & $\begin{array}{c}14 \\
(13.5)\end{array}$ & $\begin{array}{c}67 \\
(33.3)\end{array}$ & $\begin{array}{c}113 \\
(56.2)\end{array}$ & $\begin{array}{c}21 \\
(10.4)\end{array}$ \\
\hline $3-4$ & $\begin{array}{c}8 \\
(8.7)\end{array}$ & $\begin{array}{c}55 \\
(59.8)\end{array}$ & $\begin{array}{c}29 \\
(31.5)\end{array}$ & $\begin{array}{c}10 \\
(6.6)\end{array}$ & $\begin{array}{c}89 \\
(58.9)\end{array}$ & $\begin{array}{c}52 \\
(34.4)\end{array}$ & $\begin{array}{c}18 \\
(7.4)\end{array}$ & $\begin{array}{c}144 \\
(59.3)\end{array}$ & $\begin{array}{c}81 \\
(33.3)\end{array}$ \\
\hline$\geq 5$ & $\begin{array}{c}1 \\
(2.8)\end{array}$ & $\begin{array}{c}13 \\
(36.1)\end{array}$ & $\begin{array}{c}22 \\
(61.1)\end{array}$ & 0 & $\begin{array}{c}31 \\
(32.3)\end{array}$ & $\begin{array}{c}65 \\
(67.7)\end{array}$ & $\begin{array}{c}1 \\
(0.8)\end{array}$ & $\begin{array}{c}44 \\
(33.3)\end{array}$ & $\begin{array}{c}87 \\
(65.9)\end{array}$ \\
\hline \multicolumn{10}{|l|}{ Diabetes $^{\mathrm{a}}$} \\
\hline No & $\begin{array}{c}45 \\
(23.3)\end{array}$ & $\begin{array}{c}106 \\
(54.9)\end{array}$ & $\begin{array}{c}42 \\
(21.8)\end{array}$ & $\begin{array}{c}35 \\
(12.3)\end{array}$ & $\begin{array}{c}155 \\
(54.6)\end{array}$ & $\begin{array}{c}94 \\
(33.1)\end{array}$ & $\begin{array}{c}80 \\
(16.8)\end{array}$ & $\begin{array}{c}261 \\
(54.7)\end{array}$ & $\begin{array}{c}136 \\
(28.5)\end{array}$ \\
\hline Yes & $\begin{array}{c}3 \\
(9.4)\end{array}$ & $\begin{array}{c}13 \\
(40.6)\end{array}$ & $\begin{array}{c}16 \\
(50.0)\end{array}$ & $\begin{array}{c}3 \\
(4.5)\end{array}$ & $\begin{array}{c}27 \\
(40.9)\end{array}$ & $\begin{array}{c}36 \\
(54.5)\end{array}$ & $\begin{array}{c}6 \\
(6.1)\end{array}$ & $\begin{array}{c}40 \\
(40.8)\end{array}$ & $\begin{array}{c}52 \\
(53.1)\end{array}$ \\
\hline \multicolumn{10}{|l|}{ Hypertension ${ }^{\mathrm{a}}$} \\
\hline No & $\begin{array}{c}40 \\
(40.0)\end{array}$ & $\begin{array}{c}51 \\
(51.0)\end{array}$ & $\begin{array}{c}9 \\
(9.0)\end{array}$ & $\begin{array}{c}30 \\
(22.9)\end{array}$ & $\begin{array}{c}73 \\
(55.7)\end{array}$ & $\begin{array}{c}28 \\
(21.4)\end{array}$ & $\begin{array}{c}70 \\
(30.3)\end{array}$ & $\begin{array}{c}124 \\
(53.7)\end{array}$ & $\begin{array}{c}37 \\
(16.0)\end{array}$ \\
\hline Yes & $\begin{array}{c}8 \\
(6.4)\end{array}$ & $\begin{array}{c}68 \\
(54.4)\end{array}$ & $\begin{array}{c}49 \\
(39.2)\end{array}$ & $\begin{array}{c}8 \\
(3.6)\end{array}$ & $\begin{array}{c}109 \\
(49.5)\end{array}$ & $\begin{array}{c}103 \\
(46.8)\end{array}$ & $\begin{array}{c}16 \\
(4.6)\end{array}$ & $\begin{array}{c}177 \\
(51.3)\end{array}$ & $\begin{array}{c}152 \\
(44.1)\end{array}$ \\
\hline \multicolumn{10}{|c|}{ Bone and articular diseases ${ }^{a}$} \\
\hline No & $\begin{array}{c}41 \\
(25.3)\end{array}$ & $\begin{array}{c}88 \\
(54.3)\end{array}$ & $\begin{array}{c}33 \\
(20.4)\end{array}$ & $\begin{array}{c}30 \\
(18.0)\end{array}$ & $\begin{array}{c}96 \\
(57.5)\end{array}$ & $\begin{array}{c}41 \\
(24.6)\end{array}$ & $\begin{array}{c}71 \\
(21.6)\end{array}$ & $\begin{array}{c}184 \\
(55.9)\end{array}$ & $\begin{array}{c}74 \\
(22.5)\end{array}$ \\
\hline Yes & $\begin{array}{c}7 \\
(11.5)\end{array}$ & $\begin{array}{c}30 \\
(49.2)\end{array}$ & $\begin{array}{c}24 \\
(39.3)\end{array}$ & $\begin{array}{c}7 \\
(3.8)\end{array}$ & $\begin{array}{c}86 \\
(47.0)\end{array}$ & $\begin{array}{c}90 \\
(49.2)\end{array}$ & $\begin{array}{c}14 \\
(5.7)\end{array}$ & $\begin{array}{c}116 \\
(47.5)\end{array}$ & $\begin{array}{c}114 \\
(46.7)\end{array}$ \\
\hline
\end{tabular}

a $P<0.05$ in all subgroups (overall, men, and women).

b $P>0.05$ among men.

c $P<0.05$ overall.

pendent predictive factor of increased medication use (16). Older studies $(5,17)$ and more recent ones $(15,18)$ have observed that a higher proportion of older women than men are multiple users and that age associates positively with use of five or more medications $(5,15,17,18)$.

In Brazil, a population survey in a state capital in the Northeast estimated that, depending on the area, between $5 \%$ and $14 \%$ of elderly peo- ple use five or more prescribed products. To this must be added the $7 \%$ to $17 \%$ using at least two nonprescribed products (9). A population survey in a city in the Southeast estimated that $13 \%$ of the elderly use five or more 
TABLE 5. Prevalence of drug use (two strata) by health condition and health service variables, among retirees 60 years old or older, Rio de Janeiro City, Rio de Janeiro, Brazil, 2003

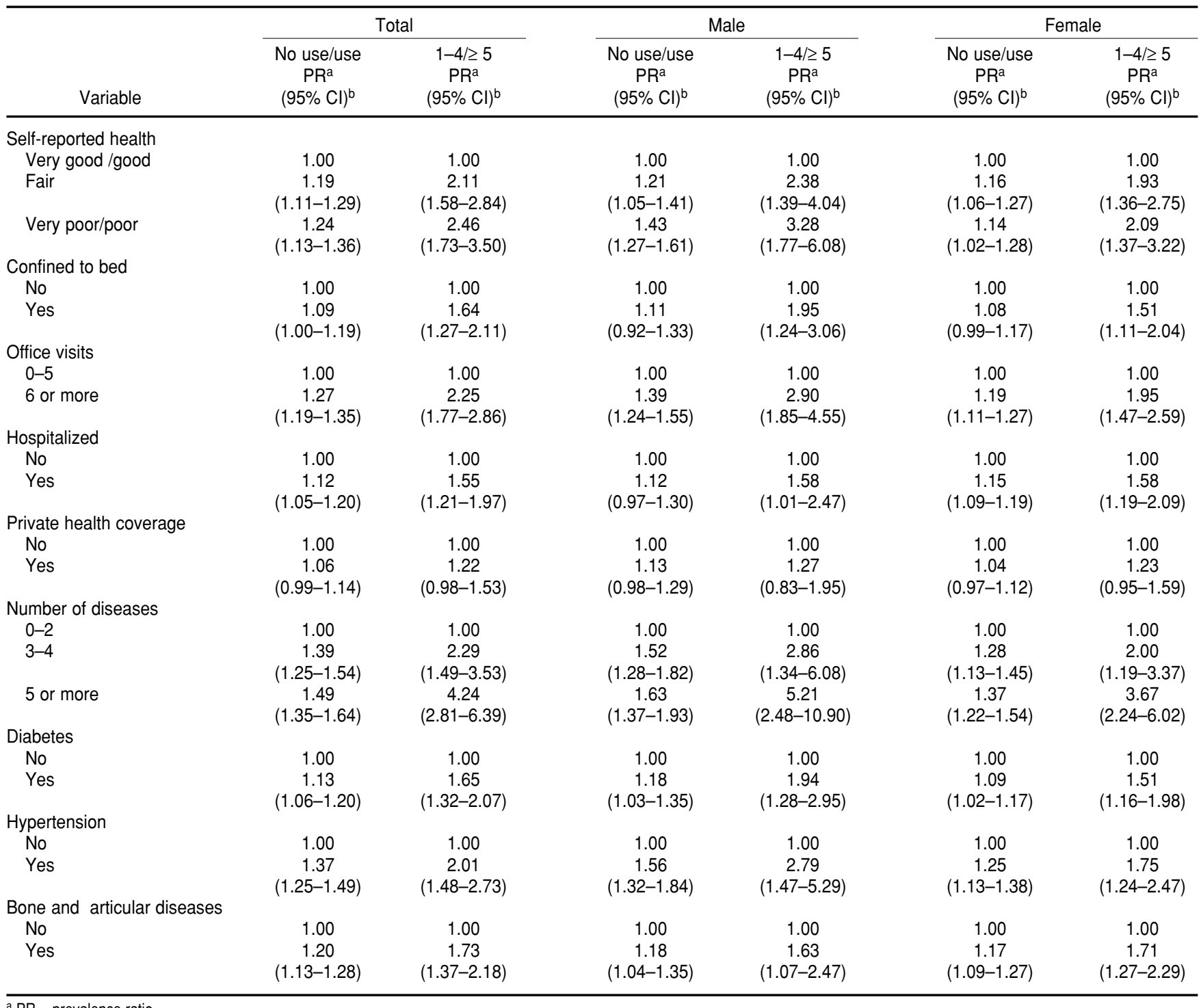

a $\mathrm{PR}=$ prevalence ratio.

${ }^{\mathrm{b}} \mathrm{Cl}=$ confidence interval.

drugs; they observed also that a higher proportion of women are multiple users $(16.9 \%$ versus $10.5 \%)$ and female gender is independently associated with use of five or more drugs (10).

The sample studied consisted of relatively young elderly [average age (standard deviation) $=72.27$ (64.580.0); median $=72.0$ ], who administer their medication independently and are not in hospitals or nursing homes. However, the number of products used varies from 1 to 24 , and $11 \%$ of the sample use 7 or more products. Therefore, estimates of multiple users must be evaluated with care.

Although the elderly consume a disproportionately large number of medications because they have multiple chronic diseases and disorders, the simultaneous use of several products is worrying, given the possibility of adverse events, the exceptional expense, and noncompliance with prescriptions for products essential to preventing and controlling chronic diseases.

Polypharmacy is not always avoidable. Diseases such as arterial hypertension and diabetes mellitus (DM), highly prevalent among the elderly, usually require the use of several products of proven efficacy. However, it must be determined whether treatment guidelines are being followed and whether patients are using only the products indicated clinically. 
TABLE 6. Potentially inappropriate medications used by retirees 60 years old or older, Rio de Janeiro City, Rio de Janeiro, Brazil, 2003

\begin{tabular}{|c|c|c|c|c|}
\hline Active substances & ATC code & No. & $\%$ & Severity rating \\
\hline Cimetidine & A02BA01 & 4 & 1.6 & Low \\
\hline Gastrointestinal antispasmodic drugs ${ }^{a}$ & A03 & 14 & 5.5 & High \\
\hline Laxatives $^{b}$ & $\mathrm{~A} 06 \mathrm{AB}$ & 7 & 2.7 & High \\
\hline Chlorpropamide & A10BB02 & 4 & 1.6 & High \\
\hline Ticlopidine & B01AC05 & 6 & 2.3 & High \\
\hline Ferrous sulfate & B03АA07 & 13 & 5.1 & Low \\
\hline Digoxin & C01AA05 & 17 & 6.6 & Low \\
\hline Amiodarone & C01BD01 & 18 & 7.0 & High \\
\hline Methyldopa & C02AB02 & 11 & 4.3 & High \\
\hline Clonidine & $\mathrm{C} 02 \mathrm{AC} 01$ & 3 & 1.2 & Low \\
\hline Doxazosin & C02CA04 & 2 & 0.8 & Low \\
\hline Ergot mesyloids & C04AE, N02CA & 12 & 4.7 & Low \\
\hline Estrogens & G03CA & 9 & 3.5 & Low \\
\hline Nitrofurantoin & G04AC01 & 1 & 0.4 & High \\
\hline Indomethacin & M01AB01 & 1 & 0.4 & High \\
\hline NSAIDSc & M01AC & 7 & 2.7 & High \\
\hline Muscle relaxants and antispasmodics & M03B, G04BD04 & 39 & 15.2 & High \\
\hline Phenytoin & N03AB02 & 5 & 2.0 & High \\
\hline Thioridazine & N05AC02 & 3 & 1.2 & High \\
\hline Benzodiazepines & N05BA & 33 & 12.9 & High \\
\hline Flurazepam & N05CD01 & 1 & 0.4 & High \\
\hline Amitriptyline & N06AA09 & 6 & 2.3 & High \\
\hline Fluoxetine & N06AB03 & 6 & 2.3 & High \\
\hline Antihistamines $^{d}$ & R06A, N05BB01 & 34 & 13.3 & High \\
\hline Total & & 256 & 100.0 & \\
\hline
\end{tabular}

a Camylofin, trimebutine, papaverine, isometheptene, alverine, hyoscyamine, belladonna alkaloids.

b Bisacodyl, cascara sagrada.

c Piroxicam, meloxicam, tenoxicam. NSAIDS = nonsteroidal anti-inflammatory drugs.

d Diphenhydramine, doxylamine, dexchlorpheniramine, chlorpheniramine, promethazine, desloratadine, cyproheptadine, loratadine, fexofenadine, hydroxyzine, pheniramine, dimetindene.

In the case of arterial hypertension, treatment should begin with nondrug therapeutic interventions. If medication must be given, it is recommended to start with one product capable of treating hypertension simultaneously with whatever other conditions are present and offering good tolerability; to start with a low dose and increase it before adding a second medication; to investigate thoroughly the causes of the hypertension if the response to two medications is unsatisfactory; and, before introducing a third medication, to reevaluate diet and sodium intake (19).

DM prevalence in the population over 65 years of age is estimated at $20 \%$. DM management should begin with medical nutrition therapy and an exercise regimen to increase insulin sensitivity and promote weight loss. If the patient's glycemic target is not achieved after 3 or 4 weeks, drug therapy is indicated. It is estimated that complications are present in only $20 \%$ to $50 \%$ of individuals with newly diagnosed type $2 \mathrm{DM}$ (20). Although DM is a progressive disease that will ultimately require multiple therapies, all patients will not need the complete therapeutic arsenal.

Of the $17 \%$ of participants $(98 / 577)$ who reported DM, half (49/98) are also hypertensive. Use of several products to control chronic diseases may be indicated for those 49 individuals. However, far more than that number (189) use five or more products. The use of products with inappropriate ingredients or to control symptoms (antiinflammatories, muscle relaxants, analgesics, cough and cold preparations), or with no clear therapeutic indication for this population (vitamins and mineral salts), may be jeopardizing the attention given to chronic diseases, such as DM, or prescription compliance with essential medications.

Our study inventoried all the products participants used. In analyzing the data, the variable "use of medications" was treated as discrete, and several different cutoff points were set. This was done to evaluate the validity of using a two-way question on medication use instead of inventorying in health surveys. We observed that the estimates obtained were generally similar, suggesting that the question "Do you use medications?" (yes or no) may be a viable alternative to inventory in large-scale surveys of health and medication use.

\section{Inappropriate use}

Of the active substances used by the sample, $10 \%$ were considered inappropriate by the criteria adopted (2), most of them severely. The most widely used substances (muscle relaxants, antihistamines, and long-acting benzodiazepines) have anticholinergic adverse effects, such as sedation, weakness, falls, 
and fractures and thus may be dangerous for this age group.

According to Fick et al. (2), a recent study estimated that the prevalence of potentially inappropriate medication in a Medicare managed-care population was $23 \%$. The patients most likely to take potentially inappropriate medications were those with higher care costs and more office visits, even after adjustment for sex, severity of the diseases, and more prescribed medications. The medications contraindicated for the elderly that were consumed most frequently were propoxyphene, amitriptyline, dipyridamole, diazepam, and chlorpropamide (2).

One Brazilian survey found between $13 \%$ and $19 \%$ of elderly people using products that contained inappropriate substances (9). Another study, of women participating in an organization at a public university in Rio de Janeiro that promotes cultural activities directed to well-being for the elderly, estimated the proportion of inappropriate substances being used at $17 \%(8)$.

Our results provided important clues to identifying possible risk factors for excessive consumption of medications. The positive associations of increasing use with diseases and with age are to be expected to some extent, given that the aging process entails an accumulation of chronic or degenerative conditions that require continuous drug therapy. Also, the more fragile constitution of the elderly may render them more prone to acute conditions requiring the use of medication for symptom relief.

Meanwhile, the associations encountered between increasing use of medication and the variables "school- ing" and "home owner," although not statistically significant, do suggest that consumption depends on a better economic or social situation. These findings are compatible with the overall profile of the study population, where substantial numbers of individuals have access to ambulatory and hospital care, both in the public system and through private health insurance.

However, as this is an exploratory study with limited statistical power, it was not possible to achieve significance in estimates relating the number of products consumed to certain economic variables or to subject the data to multivariate analysis. Studies designed specifically to address these relationships are to be conducted in the future. Such studies may shed light on the complex phenomenon of medication use and help distinguish a product's use for real health needs from use (whether under- or overuse) deriving from access factors.

A representative sample of 577 retirees receiving state pensions and aged 60 years or older were interviewed. This number corresponds to $64 \%$ of the people in this age group living in Rio de Janeiro. However, caution is needed in generalizing our results. Our sample has sex distributions and illiteracy rates comparable to those of the general population of Rio de Janeiro, but a higher proportion of people over age $80(21.3 \%$ versus $12.5 \%$ ) and of people who self-rated their health as very good or good ( $46.2 \%$ versus $41.1 \%)$, who have private health coverage $(43.1 \%$ versus $39.9 \%$ ), made six or more office visits in 1 year $(40.1 \%$ versus $30.3 \%)$, and were hospitalized in the preceding 12 months (13.9\% versus $10.8 \%$ ).
As a general rule, products containing benzodiazepines and antidepressants cannot be purchased without a prescription, which is not true of the other products. Meanwhile, substances widely used by the study participants to treat cardiovascular diseases, such as digoxin, amiodarone, methyldopa, and clonidine, are commonly prescribed by physicians.

Therefore, to improve drug therapy for the elderly, health practitioners can take measures to reduce unwarranted use of medication and to optimize the benefits of important drugs. Such measures include adhering to the guidelines; avoiding prescribing to relieve common symptoms amenable to general treatment, diet, or lifestyle changes; regularly reviewing medications; auditing prescription data; having clinical pharmacists visit physicians and patients; having computerized alert systems; and so on.

Further studies should be conducted to adjust lists of medications inappropriate for the elderly to the situation in developing countries.

Acknowledgments. The authors acknowledge the participation of Carlos Henrique Klein, Andréia Queiroz Ribeiro, and Célia Regina de Andrade in planning the research and collecting the data and of Cristiano Moura de Soares in developing the databases. This study was funded by the Pharmaceutical Services Technical Department (GTAF/DAB/SPS, under contract $796 / 2002$ ) of the Ministry of Health. The study also received support from the School of Pharmacy/ Minas Gerais Federal University and from the National School of Public Health/Oswaldo Cruz Foundation.

\section{REFERENCES}

1. Hemminki E, Heikkilä J. Elderly people's compliance with prescriptions, and quality of medication. Scand J Soc Med. 1975;3:87-92.

2. Fick DM, Cooper JW, Wade WE, Waller JL, Maclean R, Beers MH. Updating the Beers criteria for potentially inappropriate medication use in older adults. Results of a US Consensus Panel of Experts. Arch Intern Med. 2003;163: 2716-24.
3. Aparasu RR, Fliginger SE. Inappropriate medication prescribing for the elderly by office-based physicians. Ann Pharmacother. 1997;31(7-8):823-9.

4. Zhan C, Sangl J, Bierman AS, Miller MR, Friedman B, Wickizer SW, et al. Potentially inappropriate medication use in the communitydwelling elderly: findings from the 1996 Med- ical Expenditure Panel Survey. JAMA. 2001; 286(22):2823-9.

5. Anderson G, Kerluke K. Distribution of prescription drug exposures in the elderly: description and implications. J Clin Epidemiol. 1996;49(8):929-35.

6. Bertoldi AD, Barros AJD, Hallal PC, Lima RC Utilização de medicamentos em adultos: pre- 
valência e determinantes individuais. Rev Saude Publica. 2004;38(2):228-38.

7. Flores LM, Mengue SS. Uso de medicamentos por idosos em região do sul do Brasil. Rev Saude Publica. 2005;39(6):924-9.

8. Mosegui GBG, Rozenfeld S, Veras RP, Vianna CMM. Avaliação da qualidade do uso de medicamentos em idosos. Rev Saude Publica. 1999;33(5):437-44.

9. Coelho Filho JM, Marcopito LF, Castelo A Perfil de utilização de medicamentos por idosos em área urbana do nordeste do Brasil. Rev Saude Publica. 2004;38(4):557-64.

10. Loyola Filho AI, Uchoa E, Lima-Costa MFF. A population-based study on use of medication by the elderly in greater metropolitan Belo Horizonte, Minas Gerais, Brazil. Cad Saude Publica. 2006;22(12):2657-67.

11. Acurcio FA, Rozenfeld S, Ribeiro AQ, Klein $\mathrm{CH}$, Moura CS, Andrade CR. Utilização de medicamentos por aposentados brasileiros-I Metodologia e resultados de cobertura de in- quérito multicêntrico. Cad Saude Publica. 2006;22(1):87-96.

12. Dicionário de especialidades farmacêuticas, DEF 2000-2001. 29th ed. Rio de Janeiro: Publicações Científicas; 2000.

13. WHO Collaborating Centre for Drug Statistics Methodology. Anatomical therapeutic chemical-ATC classification index with defined daily doses (DDDs). Oslo: World Health Organization; 2000.

14. Simões MJS, Farache Filho A. Consumo de medicamentos em região do Estado de São Paulo (Brasil), 1985. Rev Saude Publica. 1988; 22:494-9.

15. Kaufman DW, Kelly JP, Rosenberg L, Anderson TE, Mitchell AA. Recent patterns of medication use in the ambulatory adult population of the United States-the Slone Survey. JAMA. 2002;287(3):337-44.

16. Veehof LJG, Meyboom-de Jong B, HaaijerRuskamp FM. Polypharmacy in the elderlya literature review. Eur J Gen Pract. 2000;6: 98-106.
17. Klaukka T, Mäkelä M, Sipilä J, Martikainen J. Multiuse of medicines in Finland. Med Care. 1993;31(5):445-50.

18. Roe CM, McNamara AM, Motheral BR. Use of chronic medications among a large, commercially-insured US population. Pharmacoepidemiol Drug Saf. 2002;11(4):301-9.

19. Fisher VJ, Williams GH. Hypertensive vascular disease. Harrison's principles of internal medicine. 16th ed. New York: McGraw-Hill Companies; 2005. Pp. 1463-81.

20. Powers AC. Diabetes mellitus. Harrison's principles of internal medicine. 16th ed. New York: McGraw-Hill Companies; 2005. Pp. 2152-80.

Manuscript received 9 October 2006. Revised version accepted for publication 29 November 2007.

RESUMEN Objetivos. Describir el uso de medicamentos en los jubilados brasileños, con énfasis en su uso inapropiado.

Métodos. Estudio transversal mediante entrevistas presenciales a una muestra sim-

Uso de medicamentos y polifarmacia en adultos mayores: encuesta en la ciudad de Rio de Janeiro, Brasil ple aleatoria de 800 jubilados del Instituto Brasileño de Seguridad Social, de 60 años de edad o más, que residen en la ciudad de Rio de Janeiro.

Resultados. Del total de encuestados, $85 \%$ informó el uso de medicamentos en los 15 días previos al estudio, con una media de 3,7 productos por persona (desviación estándar $=2,9$ ). Aproximadamente la mitad de los encuestados utilizaron de uno a cuatro medicamentos y una tercera parte empleó cinco o más. El mayor número de medicamentos usado por un paciente fue de 24. Más mujeres que hombres emplearon múltiples medicamentos. Se observó una tendencia hacia una asociación positiva $(P<0,001)$ entre el consumo de medicamentos y las variables relacionadas con las enfermedades y la atención sanitaria. Esta tendencia se mantuvo al hacer el análisis según el sexo. Los hombres que informaron padecer cinco enfermedades o más tuvieron cinco veces más probabilidad de usar múltiples medicamentos que los hombres con dos enfermedades o menos (razón de prevalencia $[R P]=5,21$; intervalo de confianza de 95\% [IC95\%]: 2,48 a 10,90). Las mujeres que informaron cinco enfermedades o más tuvieron casi cuatro veces más probabilidad de emplear varios medicamentos que las mujeres que padecían dos enfermedades o menos ( $R P=3,67$; IC $95 \%$ : $2,24$ a 6,02$)$. Se consideró que $10 \%$ de las substancias activas empleadas por los encuestados eran inapropiadas.

Conclusiones. Para mejorar el tratamiento de los adultos mayores con medicamentos, los médicos pueden tomar medidas tendentes a reducir el uso injustificado de medicamentos y optimizar los beneficios de los medicamentos de interés. Se deben realizar estudios para ajustar las listas de medicamentos inapropiados para los adultos mayores a las condiciones de los países en desarrollo.

Palabras clave Salud del anciano, utilización de medicamentos, polifarmacia, estudios transversales, Brasil. 